\title{
Systematic Molecular Dynamics Searching in a Lipid Bilayer: Application to the Glycophorin A and Oncogenic ErbB-2 Transmembrane Domains
}

\author{
Andrew J. Beevers and Andreas Kukol * \\ Department of Biological Sciences, University of Warwick, Coventry, UK CV4 7AL
}

\begin{abstract}
Molecular dynamics (MD) simulations of proteins in a lipid bilayer environment are usually undertaken with one or a few starting structures. Here we report a search protocol for systematically exploring the possible interactions in helical bundle transmembrane proteins, a frequently occurring structural motif. The search protocol correctly identifies the experimentally known structure of the dimeric human glycophorin A transmembrane domain as the lowest energy structure among five different models without any prior assumptions, whilst an identical in vacuo search fails to identify the correct structure. The lowest energy structure from the search in a lipid bilayer has a root mean square deviation of $1.1 \AA$ to the experimental structure. We have applied the same search protocol to the unknown transmembrane structure of the oncogenic mutant ErbB-2 protein, a member of the family of epidermal growth factor receptors. Resulting structures show the role of glutamic acid hydrogen bonding and close helical packing. Water molecules may also play a key role in stabilisation of the transmembrane helix association.
\end{abstract}




\section{Keywords}

Neu, Membrane Proteins, Dimerisation, Helical Packing, Hydrogen Bonding, Molecular Modelling

\section{Introduction}

In contrast to the large number of known soluble protein structures, very few transmembrane proteins have been fully structurally resolved. Apart from the problem to obtain membrane proteins in sufficient quantities, solution-state NMR spectroscopy faces the problem of the large size of protein/lipid aggregates, while x-ray crystallography needs high quality diffracting crystals. Membrane proteins prove difficult to crystallise due to their tendency to aggregate non-specifically and the requirement for detergents carries a high risk that these chemicals will interfere with the native structure of the protein complex. With the recent advances in computer power, molecular modelling of proteins in lipid bilayers has found increased attention in an attempt to gain an insight into transmembrane protein structure [1].

One of the most difficult problems, and yet also one of the most important for any modeller, is to ensure that a sufficient area of the potential energy hyper-surface of a system has been explored by the simulation. In molecular terms, this means that all possible conformations of the model should be encountered in the simulation. Even with long time molecular dynamics (MD) simulations this is only possible for very small molecules with a few degrees of freedom. Larger systems, of course, have exponentially greater numbers of possible arrangements, many of which are extremely energetically unstable. However, increasing the number of starting states and systematically varying the position of those states on the energy hyper-surface 
allows us to extensively explore all possible energetically acceptable arrangements of the structure.

This has been implemented for symmetrical $\alpha$-helical transmembrane oligomers, by systematically varying the helix rotation angle thus exploring all possible interactions between transmembrane helices in vacuo [2, 3]. However, only in connection with other experimental data has this in vacuo simulation lead to accurate experimental structures e.g. for the M2 proton channel of the Influenza A virus [4], the CM2 transmembrane domain of the Influenza $\mathrm{C}$ virus [5] or the MHC associated invariant chain [6]. Site specific infrared dichroism has been proven to successfully provide structural data, which can be used as additional energy constraints to improve the in vacuo molecular dynamics simulations [7]. In vacuo MD ignores the interaction of explicit lipid molecules with the protein and also the effect of water molecules and ions added to neutralise the charges of the protein.

Here we report the development of a search protocol, which applies MD simulations to transmembrane helical bundles created with systematic variation of their rotation angles, exploring inter-helical interactions within a lipid bilayer/water system. We validate this method with the transmembrane domain of Glycophorin A, a human sialoglycoprotein, abundantly found in erythrocytes [8, 9]. The structure of the transmembrane homodimeric complex is known by NMR $[10,11]$ and can be obtained from the protein databank [12].

We apply this method to ErbB-2, a member of the family of epidermal growth factor receptors [13], which are involved in mediating cell growth and differentiation [14]. ErbB receptors are composed of an extracellular ligand binding region, a transmembrane region and a cytoplasmic tyrosine kinase. Activation of the tyrosine kinase occurs by ligand induced dimerisation, a generally accepted activation pathway 
for all receptor tyrosine kinases [15]. The ErbB-2 receptor in particular is activated by heteromeric association with other ErbB receptors which explains the fact that no high affinity ligand for ErbB-2 has been found [16]. The oncogene neu encodes a mutant ErbB-2 receptor, which contains a single Val-Glu mutation at position 664 in the transmembrane domain [17]. This mutation causes permanent association and activation of ErbB-2 that leads finally to tumour formation [18, 19]

NMR and infrared spectroscopic studies of the mutant ErbB-2 have shown that the glutamic acid residue is protonated and strongly hydrogen bonded. This has lead to a model of the transmembrane dimer stabilised by symmetric hydrogen bonding between the carboxyl groups of protonated glutamic acid residues [20]. However, in vacuo energy minimisation conformational searches and molecular dynamics simulations have shown that this mode of interaction is incompatible with tight helical packing implying a different mode of dimer stabilisation [21, 22].

Here we systematically explore molecular interactions of different rotations of the transmembrane helical dimer of mutant ErbB-2 by MD simulations in an explicit lipid bilayer environment leading to possible models of the transmembrane structure.

\section{Methodology}

An overview of the procedure is given in the flowchart in figure 1.

\section{Generation of Peptide Helical Oligomers}

In this study, the transmembrane peptide segments of both the mutant ErbB-2 protein (residues 652-684 RASPVTFIIATVEGVLLFLILVVVVGILIIKRRR) with protonated glutamic acid and the Glycophorin A protein (residues 70-96 EPEITLIIFGVMAGVIGTILLISYGIR) and were used. As starting structures helical dimers were generated using a modified version of the CHI suite of programs for 
CNS-SOLVE [23-25], rotating the helices symmetrically through $360^{\circ}$ in increments of $10^{\circ}$ for left and right-handed dimers with crossing angles of $+25^{\circ}$ and $-25^{\circ}$ respectively, thus producing 72 different structures. For each rotation the structure was produced four times, in order to perform MD simulations in a lipid bilayer with various random initial atom velocities, giving a total of 288 starting structures. Note than an asymmetric search protocol would produce 36 x 288 structures, which we considered as computationally too expensive, while previous studies have indicated that the symmetric search is sufficient for $\alpha$-helical oligomers [2]. Individual CHI programs have been modified in order to remove the in vacuo MD simulations, while the interface to the GROMACS software is provided by in-house developed programs, which are available on request.

\section{Lipid bilayer MD simulations}

We obtained a lipid bilayer with 128 DMPC molecules, pre-equilibrated with 20ns of MD [26] and removed four to six DMPC molecules from the centre, which had a maximum distance to the peptide structure of $0.03 \AA$ (any water molecules present within this radius were also removed). Then a cylindrical hole of $1.6 \mathrm{~nm}$ radius was created during 30 ps of MD simulation using an outwards directed force on the lipid molecules [27]. Each peptide starting structure was inserted into this hole after removing overlapping lipid and water molecules (exclusion distance of $0.02 \AA$ ). After addition of counterions to neutralise the charge, the system was subjected to 100 ps of MD simulation with position restraints on the peptide molecule to allow equilibration of the surrounding lipid and water molecules, similar to other studies which used 50ps equilibration time [27]. Then the system was energy minimised and subjected to 200 ps unrestrained MD simulations at $298 \mathrm{~K}$ followed by another energy minimisation. 
The simulations were conducted with periodic boundary conditions, Berendsen temperature coupling and pressure coupling [28] and bond-lengths were constrained with the LINCS algorithm allowing a time step of 2 fs [29]. Electrostatic interactions were treated with a twin-range cut-off method of $1.8 \mathrm{~nm} / 1.0 \mathrm{~nm}$ and Van-der-Waal's interactions were calculated with a cut-off radius of $1.0 \mathrm{~nm}$. Other more exact treatments of electrostatic interactions exist, e.g. Ewald mesh methods, which may induce artificial periodicity that may influence peptide conformation [30], but we chose the faster twin-range cut-off method allowing us to carry out MD simulation of 288 structures within reasonable time. However, we used a higher cut-off radius of $1.8 \mathrm{~nm}$ compared to $1.7 \mathrm{~nm}$ in comparable lipid bilayer studies [31]..

All MD simulations were conducted with the GROMACS software [32, 33] using the GROMOS-87 forcefield [34] modified with additional forcefield parameters for lipid molecules [35, 36] (later versions of this forcefield do not currently possess parameters for lipid molecules). Simulations were performed in parallel on an 8-node dual processor LINUX cluster with Intel Xeon 2.66 GHz processors (Streamline Computing, Warwick UK).

\section{Clustering of Structures}

Clusters of similar protein structures were identified based on the criterion that the root mean square deviation (RMSD) of C $\alpha$ atoms was equal or less than $2 \AA$ between each of the cluster members and at least 10 structures contributed to each cluster. For each cluster an average structure was calculated. Each averaged structure was inserted into a DMPC bilayer as described above and the same simulation procedure described above was performed on each system, but extending the unrestrained MD simulation time to 500 ps. 


\section{Analysis of Output}

After simulation of the average structures, each resulting model was analysed according to the energy arising from non-covalent interactions including interactions with lipid and water molecules, crossing angle and rotation angle. RMSD comparisons of the experimental structure (protein data bank number: 1AFO.pdb) and MD simulated models of Glycophorin A were performed using the SWISS-PDB viewer [37]. Rotational plots were produced using the Mathematica software [38]. Images of the structures were created using the VMD molecular viewer [39].

\section{Results and Discussion}

\section{Dimer of Glycophorin A}

After 200 ps MD simulation of the 288 structures (figure 2) 7 clusters of similar structures were found and calculated cluster average structures were again subjected to a longer MD simulation of 500 ps followed by energy minimisation. The resulting average structures are also plotted in figure 2 with respect to energy and helix rotation. Encouragingly the lowest energy cluster structure 3 gives a backbone RMSD of $1.1 \AA$ to the experimentally resolved NMR structure. This structure has a helix rotation angle of $36^{\circ}$ with respect to starting structure, the helices cross right-handed with an average tilt relative to the bilayer normal of $17^{\circ}$ (Table 1). The measured crossing angle was $31.2^{\circ}$, similar to that observed in lipid bilayers [11]. The bulky aromatic residues are on the exterior of the helix dimer, minimising steric clashes. Hydrogen bonding interactions occur between threonine residues at position 87 and backbone carbonyl oxygen atoms. The methionine groups protrude into the lipid environment, whilst valine residues form a groove at the interface allowing for close 
packing of the glycine residue on the opposite helix at the dimer interface as previously shown by NMR spectroscopy [10]. Three other clusters exist with similar rotation, crossing and tilt angles to the lowest energy cluster, but these appear to lack the tight packing seen in the global minimum. These two clusters were created from a greater number of structures than the global minimum, containing a wider range of rotational angles. It is possible that because of this the averages created in these cases were less able to pack tightly during MD simulation (Table 1, Figure 2). This finding also demonstrates the importance of the tight glycine packing in the stability of the experimental structure, as previously indicated [10].

By contrast, the same in vacuo search protocol revealed the lowest energy cluster average structure at a completely different angle of rotation and a backbone RMSD of $3.92 \AA$ to the experimental structure. A structure similar to the NMR structure was found at a rotation similar to that of the lipid search global minimum, possessing a backbone RMSD of $0.87 \AA$; but this structure was significantly higher in energy (data not shown). Therefore it can be deduced from this work that helical searching protocols require an explicit description of the surrounding medium. The effect of favourable and unfavourable lipid-peptide molecular interactions and the steric hindrance caused by the lipid chains themselves will to a degree contribute to the stability of each possible structure. A recent study highlights this, showing that the Glycophorin A NMR structure adopts varying tilts and crossing angles in a variety of lipid types following long MD simulations [40].

\section{ErbB-2 dimer}

The lipid search protocol yielded three specific low energy structures (Table 2, Figure 3) at rotation angles of $10^{\circ}, 161^{\circ}$ and $260^{\circ}$. The two lowest energy structures have 
helical crossing angles of approximately $40^{\circ}$, whereas the third lowest has a crossing angle of $38^{\circ}$. All three structures possessed a helix tilt of approximately $20^{\circ}$ with respect to the bilayer normal.

Two structures, cluster 3 and cluster 4, involved protrusion of the protonated glutamic acid residues into the surrounding lipid environment (Figure $4 \mathrm{~b}$ and $4 \mathrm{c}$ ). This 'protruding' arrangement is contrary to that previously predicted [20] and it is unusual that polar side-chains are immersed in the lipid membrane. These particular rotations appear to satisfy packing constraints at the helical interface, as there is no significant distortion in the monomers. It is also possible for the polar glutamic acid residues to be stabilised by interaction with water molecules at the bilayer interface and with polar lipid head groups. The lowest energy structure (cluster 3) shows tight packing throughout the entire structure, whereas the packing in cluster 4 is to a certain extent lost in the glutamic acid region of the peptide. At the particular rotation of cluster 3 , threonine and serine residues are able to participate in inter-helical hydrogen bonding interactions, possibly resulting in tighter packing and adding a greater degree of stability to this complex. It also appears that with this structure, although the phenylalanine groups are at the interface of the dimer, the adopted rotation of the helices allows for favourable packing of these residues. Many structures with similar starting rotational angles are likely to have adopted this final structure after MD simulation because their starting position would have caused steric clashes. The fact that this cluster contained a larger number of structures than any other cluster strengthens this hypothesis further (Figure 3).

In the other low energy structure (cluster 1 - Figure $4 a$ ), the rotation of the peptide helices was such that the protonated glutamic acid residues were both located in contact faces of the helices, and hence able to interact. In this arrangement the 
protonated glutamic acid residues are present in or directly next to the dimer interface of the complex. Close examination of this area of the model shows possible interaction between the glutamic acid of one peptide and the carbonyl oxygens of the opposite peptide backbone whilst the other glutamic acid residue interacts with carbonyl oxygens on its own backbone (Figure 4a.). This hydrogen bonding interaction appears to be dictated to a degree by packing constraints such that the inter-helical interacting glutamic acid residue is able to sit in a 'pocket' created by leucine residues and the other glutamic acid residue. Inter-helical packing in this cluster also appears to be relatively uniform except that in this case the packing appears tightest around the glutamic acid region of the peptide and is less tight in other areas.

Existing models point to two types of inter-helical hydrogen bonding interaction, directly between the two carboxyl groups of glutamic acid side-chains [20] or between glutamic acid residues and carbonyl oxygens on the peptide backbone itself [20, 21, 41]. In the case of this study, it is elements of the latter that have been observed using this search method, in that there is one inter-helical glutamic acid to carbonyl oxygen interaction observed in the final structure. However, it was observed during the MD simulation that two interactions existed for short periods of time (data not shown), indicating that the glutamic acid residues have considerable flexibility at the helical interface. A direct Glu-Glu interaction, which was not observed, is likely to result in the loss of a large portion of the helix packing, particularly in the glutamic acid region of the peptide and recent studies have suggested that this is not the interhelical interaction observed in ErbB-2 [21, 22, 42]. Long MD simulations of selected rotations of the similar mutated human ErbB-2 transmembrane segment with glutamic acid residues at the helix interface, run for several nanoseconds in a lipid bilayer, have 
suggested that the formation of Glu-Glu hydrogen bonds may be restricted by the bilayer environment [43].

Another important observation is the ability of individual water molecules to move from the water phase into the area of the transmembrane region of the ErbB-2 peptide, and interact with glutamic acid or other polar residues, as shown in Figure 5 (no water molecules were present in the bilayer region prior to MD simulation). This effect may be a factor in reducing the affinity of direct Glu-Glu and Glu-backbone hydrogen bonds. The possible interaction of water with protonated glutamic acid in this environment has previously been suggested by long MD simulations of the mutated human ErbB-2 transmembrane peptide in an explicit lipid bilayer [43, 44]. Recent work has also suggested that water may be a key factor in the dimerisation process of the mutant ErbB-2 domain [45].

Unlike Glycophorin A, the atomic structure of the mutant ErbB-2 transmembrane domain is not known but only a limited set of structural aspects. The key observation here is the presence of glutamic acid hydrogen bonds and the close proximity of these residues to the interface between the helices [20]. Based on this data, it would appear that cluster 1 is the closest to the experimental structure, although this is not the global minimum. Also, recent studies have indicated that small and/or polar residues are more likely to occur in helical interfaces than other amino acids [46]. However, the presence of several energetically stable structural arrangements would indicate that glutamic acid H-bonding is not the sole factor in the determination of inter-helical association and other factors can compensate for the loss of this particular interaction. The Glycophorin A structure can be correctly deduced from this method, which is due to the well defined GXXXG packing motif in the transmembrane domain [9, 47], 
whereas in mutant ErbB-2 numerous inter-helical associations lead to energy minima and MD alone is insufficient to distinguish between them.

\section{Conclusion}

This work reports the development of a systematic search of symmetrical transmembrane helical bundles in an explicit lipid bilayer environment. While parameters such as translation along the helical axis and inter-helical separation are not systematically explored, they are allowed to vary during unrestrainded MD simulation. Certain effects, such as the initial association of the helices and the subsequent lateral movement of the oligomeric complex in the bilayer, occur over many nanoseconds [48] and therefore cannot be simulated in the time-frames used in this study. The focus of this study is therefore on inter-helical interactions and the relative energetic comparisons of differing helical rotations.

This method accurately predicts the structure of Glycophorin A without any assumptions, while previous similar in vacuo approaches have relied on experimental data [3].

We have applied this new method to the transmembrane domain of the mutant ErbB-2 dimer. For this search we have obtained several possible structures, one of which displays interactions similar to those previously predicted.. The possible role played by water molecules also highlights the necessity to include an explicit lipid/water system in these searches. However, it appears that a definite answer about the structure of the mutant ErbB-2 transmembrane domain would require additional experimental data about helix rotation and tilt angle as it can be obtained by site specific infrared dichroism [7]. 


\section{References}

1. Domene, C., P.J. Bond, and M.S.P. Sansom, Membrane Protein Simulations: Ion Channels and Bacterial Outer Membrane Proteins. Advances in Protein Chemistry, 2003. 66: p. 159-193.

2. Adams, P.D., I.T. Arkin, D.M. Engelman, and A.T. Brünger, Computational searching and mutagenesis suggest a structure for the pentameric transmembrane domain of phospholamban. Structural Biology, 1995. 2(2): p. 154-162.

3. Adams, P.D., D.M. Engelman, and A.T. Brunger, Improved Prediction for the Structure of the Dimeric Transmembrane Domain of Glycophorin A Obtained Through Global Searching. Proteins: Structure, Function and Genetics, 1996. 26: p. 257-261.

4. Kukol, A., P.D. Adams, L.M. Rice, A.T. Brunger, and I.T. Arkin, Experimentally Based Orientational Refinement of Membrane Protein Models: A Structure for the Influenza A M2 H+ Channel. Journal of Molecular Biology, 1999. 286: p. 951-962.

5. Kukol, A., J. Torres, and I.T. Arkin, Use of a single glycine residue to determine tilt and orientation of a transmembrane helix. A new structural label for infrared spectroscopy. Biophysical Journal, 2000. 79(6): p. 3139-3143.

6. Kukol, A., J. Torres, and I.T. Arkin, A Structure for the trimeric MHC Class II-associated Invariant Chain Transmembrane Domain. Journal of Molecular Biology, 2002. 320: p. 1109-1117.

7. Kukol, A., Site-specific IR spectroscopy and molecular modelling combined towards solving transmembrane protein structure. Spectroscopy, 2005. 19(1): p. 1-16.

8. Bormann, B.J., W.J. Knowles, and V.T. Marchesi, Synthetic Peptides Mimic the Assembly of Transmembrane Glycoproteins. The Journal of Biological Chemistry, 1989. 264(7): p. 4033-4037.

9. Lemmon, M.A., J.M. Flanagan, H.R. Treutlein, J. Zhang, and D.M. Engelman, Sequence Specificity in the Dimerization of Transmembrane Alpha-Helices. Biochemistry, 1992. 31: p. 12719-12725.

10. MacKenzie, K.R., J.H. Prestegard, and D.M. Engelman, A transmembrane helix dimer: structure and implications. Science, 1997. 276: p. 131-133.

11. Smith, S.O., D. Song, S. Shekar, M. Groesbeek, M. Ziliox, and S. Aimoto, Structure of the Transmembrane Dimer Interface of Glycophorin A in Membrane Bilayers. Biochemistry, 2001. 40(22): p. 6553-6558.

12. Berman, H.M., J. Westbrook, Z. Feng, G. Gilliland, T.N. Bhat, H. Wessig, I.N. Shindyalov, and B. P.E., The Protein Data Bank. Nucleic Acids Research, 2002. 28(1): p. 235-242.

13. Leahy, D.J., Structure and Function Of The Epidermal Growth Factor (EGF/ErbB) Family Of Receptors. Advances in Protein Chemistry, 2004. 68: p. 1-27.

14. Olayioye, M.A., R.M. Neve, H.A. Lane, and N.E. Hynes, The ErbB signalling network: receptor heterodimerization in development and cancer. EMBO Journal, 2000. 19(13): p. 3159-3167.

15. Schlessinger, J., Cell Signalling by Receptor Tyrosine Kinases. Cell, 2000. 103: p. 211-225.

16. Klapper, L.H., S. Glathe, N. Vaisman, N.E. Hynes, G.C. Andrews, M. Sela, and Y. Yarden, The ErbB-2/HER2 oncoprotein of human carcinomas may 
function solely as a shared coreceptor for multiple stroma-derived growth factors. Proceedings of the National Academy of Sciences USA, 1999. 96: p. 4995-5000.

17. Bargmann, C.I., M.-C. Hung, and R.A. Weinberg, Multiple independent activations of the neu oncogene by a point mutation altering the transmembrane domain of p185. Cell, 1986. 45: p. 649-657.

18. Bargmann, C.I. and R.A. Weinberg, Oncogenic activation of the neu-encoded receptor protein by point mutation and deletion. EMBO Journal, 1988. 7: p. 2043-2052.

19. Bargmann, C.I. and R.A. Weinberg, Increased Tyrosine Kinase Activity Associated with the Protein Encoded by the Activated neu Oncogene. Proceedings of the National Academy of Sciences USA, 1988. 85(15): p. 5394-5398.

20. Smith, S.O., C.S. Smith, and B.J. Bormann, Strong hydrogen bonding interactions involving a buried glutamic acid in the transmembrane sequence of the neu/erbB-2 receptor. Nature Structural Biology, 1996. 3(3): p. 252-258.

21. Sajot, N. and M. Genest, Structure prediction of the dimeric neu/ErbB-2 transmembrane domain from multi-nanosecond molecular dynamics simulations. European Biophysics Journal, 2000. 28: p. 648-662.

22. Aller, P., L. Voiry, N. Garnier, and M. Genest, Molecular Dynamics (MD) Investigations of Preformed Structures of the Transmembrane Domain of the Oncogenic Neu Receptor in a DMPC Bilayer. Biopolymers, 2005. 77: p. 184197.

23. Brunger, A.T., P.D. Adams, M. Clore, W.L. DeLano, P. Gros, R.W. GrosseKunsteleve, J.S. Jiang, J. Kuszewski, M. Nilges, N.S. Pannu, R.J. Read, L.M. Rice, T. Simonson, and G.L. Warren, Crystallography \& NMR System: A New Software Suite for Macromolecular Structure Determination. Acta Crystallographa, 1998. D54: p. 905-921.

24. Adams, P.D., N.S. Pannu, R.J. Read, and A.T. Brunger, Cross-validated maximum likelihood enhances crystallographic simulated annealing refinement. Proceedings of the National Academy of Sciences USA, 1997. 94: p. 5018-5023.

25. Brunger, A.T., P.D. Adams, and L.M. Rice, New applications of simulated annealing in X-ray crystallography and solution NMR. Structure, 1997. 5: p. 325-336.

26. Gurtovenko, A.A., M. Patra, M. Karrttunen, and I. Vattulainen, Cationic DMPC/DMPAT Lipid Bilayers: Molecular Dynamics Study. Biophysical Journal, 2004. 86: p. 3461-3472.

27. Faraldo-Gomez, J.D., G.R. Smith, and M.S.P. Sansom, Setting up and optimisation of membrane protein simulations. European Biophysical Journal, 2002. 31: p. 217-227.

28. Berendsen, H.J.C., J.P.M. Postma, W.F. Van Gunsteren, A. DiNola, and J.R. Haak, Molecular Dynamics with coupling to an external bath. Journal of Chemical Physics, 1984. 81(8): p. 3684-3690.

29. Hess, B., H. Bekker, H.J.C. Berendsen, and G.E.M. Fraajie, LINCS: A Linear Constraint Solver for Molecular Simulations. Journal of Computational Chemistry, 1997. 18(12): p. 1463-1472.

30. Weber, W., P.H. Hunenburger, and J.A. McCammon, Molecular Dynamics Simulations of a Polyalanine Octapeptide under Ewald Boundary Conditions: 
Influence of Artificial Periodicity on Peptide Conformation. Journal of Physical Chemistry B, 2000. 104(15): p. 3668-3675.

31. Law, R.J., D.P. Tieleman, and M.S. Sansom, Pores formed by the nicotinic receptor m2delta peptide: a molecular dynamics study. Biophysical Journal, 2003. 84(1): p. 14-27.

32. Lindahl, E., B. Hess, and D. van der Spoel, Gromacs 3.0: A package for molecular dynamics simulation and trajectory analysis. J. Mol. Mod., 2001. 7: p. 306-317.

33. Berendsen, H.J.C., D. van der Spoel, and R. van Drunen, GROMACS: A message passing parallel molecular dynamics implementation. Comp. Phys. Comm., 1995. 91: p. 43-56.

34. Van Gunsteren, W.F. and H.J.C. Berendsen, Gromos-87 Manual. 1987.

35. Tieleman, D.P. and H.J.C. Berendsen, Molecular dynamics simulations of a fully hydrated dipalmitoylphosphatidylcholine bilayer with different macroscopic boundary conditions and parameters. Journal of Chemical Physics, 1996. 105(11): p. 4871-4880.

36. Berger, O., O. Edholm, and F. Jahnig, Molecular Dynamics simulations of a fluid bilayer of dipalmitoylphosphatidylcholine at full hydration, constant pressure and constant temperature. Biophysical Journal, 1997. 72(5): p. 20022013.

37. Guex, N., A. Diemand, and M.C. Peitsch, Protein Modelling for all. Trends in Biochemical Sciences, 1999. 24: p. 364-367.

38. Wolfram, S., The Mathematica Book. 3 ed. 1996: Wolfram Media / Cambridge University Press.

39. Humphrey, W., A. Dalke, and K. Schulten, VMD: Visual Molecular Dynamics. Journal of Molecular Graphics, 1996. 14(1): p. 33-38.

40. Petrache, H.I., A. Grossfield, K.R. MacKenzie, D.M. Engelman, and T.B. Woolf, Modulation of Glycophorin A Transmembrane Helix Interactions by Lipid Bilayers: Molecular Dynamics Calculations. Journal of Molecular Biology, 2000. 302: p. 727-746.

41. Sternberg, M.J.E. and W.J. Gullick, Neu Receptor Dimerization. Nature, 1989. 339: p. 587.

42. Smith, S.O., C.S. Smith, S. Shekar, O. Peersen, M. Ziliox, and S. Aimoto, Transmembrane Interactions in the Activation of the Neu Receptor Tyrosine Kinase. Biochemistry, 2002. 41: p. 9321-9332.

43. Garnier, N., S. Crouzy, and M. Genest, Molecular Dynamics Simulations of the Transmembrane Domain of the Oncogenic ERbB2 Receptor Dimer in a DMPC Bilayer. Journal of Biomolecular Structure and Dynamics, 2003. 21(2): p. 179-199.

44. Soumana, O.S., P. Aller, N. Garnier, and M. Genest, Transmembrane Peptides from Tyrosine Kinase Recptor. Mutation-related behaviour in a Lipid Bilayer Investigated by Molecular Dynamics Simulations. Journal of Biomolecular Structure and Dynamics, 2005. 23(1): p. 91-99.

45. van der Ende, B.M., F.J. Sharom, and J.H. Davis, The transmembrane domain of Neu in a lipid bilayer: molecular dynamics simulations. European Biophysics Journal, 2004. 33: p. 596-610.

46. Eilers, M., A.B. Patel, W. Liu, and S.O. Smith, Comparison of Helix Interactions in Membrane and Soluble Proteins. Biophysical Journal, 2002. 82: p. 2720-2736. 
47. Lemmon, M.A., H.R. Treutlein, P.D. Adams, A.T. Brunger, and D.M.

Engelman, A dimerization motif for transmembrane alpha-helices. Nature Structural Biology, 1994. 1(3): p. 157-163.

48. Henin, J., A. Pohorille, and C. Chipot, Insights into the Recognition and Association of Transmembrane alpha-helices. The Free Energy of alpha-helix Dimerization in Glycophorin A. Journal of the American Chemical Society, 2005. 127(23): p. 8478-8484. 
Table 1: Orientational data and energies for cluster averages of Glycophorin A. The lowest energy structure (cluster 3) is indicated in bold.

\begin{tabular}{cccccc}
$\begin{array}{c}\text { Glycophorin } \\
\text { A Cluster }\end{array}$ & $\begin{array}{c}\text { Number of } \\
\text { Structures }\end{array}$ & $\begin{array}{c}\text { Rotational } \\
\text { Angle }\end{array}$ & $\begin{array}{c}\text { Crossing } \\
\text { Angle }\end{array}$ & Tilt Angle & $\begin{array}{c}\text { Energy / KJ } \\
\text { mol }^{-1}\end{array}$ \\
\hline 1 & 12 & $356.7^{\circ}$ & $29.8^{\circ}$ & $20.3^{\circ}$ & -642.1 \\
2 & 12 & $212.1^{\circ}$ & $20.6^{\circ}$ & $11.9^{\circ}$ & -626.0 \\
3 & $\mathbf{1 1}$ & $\mathbf{3 6 . 2 ^ { \circ }}$ & $-\mathbf{3 1 . 2 ^ { \circ }}$ & $\mathbf{1 7 . 4}^{\circ}$ & $\mathbf{- 6 7 1 . 6}$ \\
4 & 15 & $41.2^{\circ}$ & $-37.4^{\circ}$ & $17.4^{\circ}$ & $-621.2^{\mathrm{a}}$ \\
5 & 16 & $31.0^{\circ}$ & $-31.8^{\circ}$ & $20.6^{\circ}$ & $-621.1^{\mathrm{a}}$ \\
6 & 12 & $136.3^{\circ}$ & $-27.6^{\circ}$ & $14.4^{\circ}$ & -621.4 \\
7 & 12 & $317.1^{\circ}$ & $-31.2^{\circ}$ & $12.4^{\circ}$ & -611.7 \\
\hline
\end{tabular}

a These structures have similar rotation, tilt and crossing angles to cluster 3 but packing is not as tight.

Table 2: Orientational data and energies for cluster averages of mutant ErbB-2 protein, the three lowest energy structures are indicated in bold.

\begin{tabular}{cccccc}
$\begin{array}{c}\text { Erb-B2 } \\
\text { Cluster }\end{array}$ & $\begin{array}{c}\text { Number of } \\
\text { Structures }\end{array}$ & $\begin{array}{c}\text { Rotational } \\
\text { Angle }\end{array}$ & $\begin{array}{c}\text { Crossing } \\
\text { Angle }\end{array}$ & Tilt Angle & $\begin{array}{c}\text { Energy / KJ } \\
\text { mol }^{-1}\end{array}$ \\
\hline $\mathbf{1}$ & $\mathbf{1 2}$ & $\mathbf{1 0 . 7}^{\circ}$ & $\mathbf{3 9 . 4 ^ { \circ }}$ & $\mathbf{2 2 . 7}^{\circ}$ & $-\mathbf{3 6 1 . 9}$ \\
2 & 12 & $44.8^{\circ}$ & $27.6^{\circ}$ & $17.3^{\circ}$ & -199.8 \\
$\mathbf{3}$ & $\mathbf{1 6}$ & $\mathbf{1 6 1 . 9}^{\circ}$ & $\mathbf{4 3 . 2}^{\circ}$ & $\mathbf{2 0 . 1}^{\circ}$ & $\mathbf{- 4 0 6 . 5}$ \\
$\mathbf{4}$ & $\mathbf{1 2}$ & $\mathbf{2 6 0 . 1}^{\circ}$ & $\mathbf{2 9 . 2}^{\circ}$ & $\mathbf{2 1 . 6}^{\circ}$ & $-\mathbf{3 4 1 . 1}$ \\
5 & 12 & $105.5^{\circ}$ & $-38.4^{\circ}$ & $21.1^{\circ}$ & -177.7 \\
6 & 12 & $189.1^{\circ}$ & $-27.8^{\circ}$ & $14.2^{\circ}$ & -127.2 \\
7 & 12 & $163.6^{\circ}$ & $-29.4^{\circ}$ & $13.3^{\circ}$ & -262.9 \\
\hline
\end{tabular}

a Closest to currently available experimental data [20] 
Figure 1: Flowchart giving an overview of the search protocol employed. Only the major simulation steps are shown, leaving out energy minimisation steps and data analysis.

Figure 2A: Polar plot of the energies of structures obtained from glycophorin A MD simulations in dependence of the helix rotation angle $\phi$. The distance from the centre indicates negative energy (E) in $\mathrm{kJ} / \mathrm{mol}$. Each individual structure is indicated by a triangle, while cluster average structures are shown as numbered circles. The arcs represent the movement of structures with respect to the helix rotation angle $\phi$ during the simulation.

B: The structure defining the helix rotation $\phi=0^{\circ}$ is shown in the helical wheel diagram.

Figure 3: Polar plot of the energies of structures obtained from ErbB-2 MD simulations in dependence of the helix rotation angle $\phi$. For explanations see fig 1.

Figure 4: The three lowest energy structures (cluster averages) from the ErbB-2 simulation highlighting glutamic acid 664 in the right panel. (a) cluster 1, (b) cluster 3, (c) cluster 4.

Figure 5: The hydrogen bonding arrangements around the glutamic acid 664 residues in cluster 1 following MD simulation. Water molecules enter the bilayer region and interact with protonated Glutamic acid. Glu residues and interacting water molecules are highlighted. 


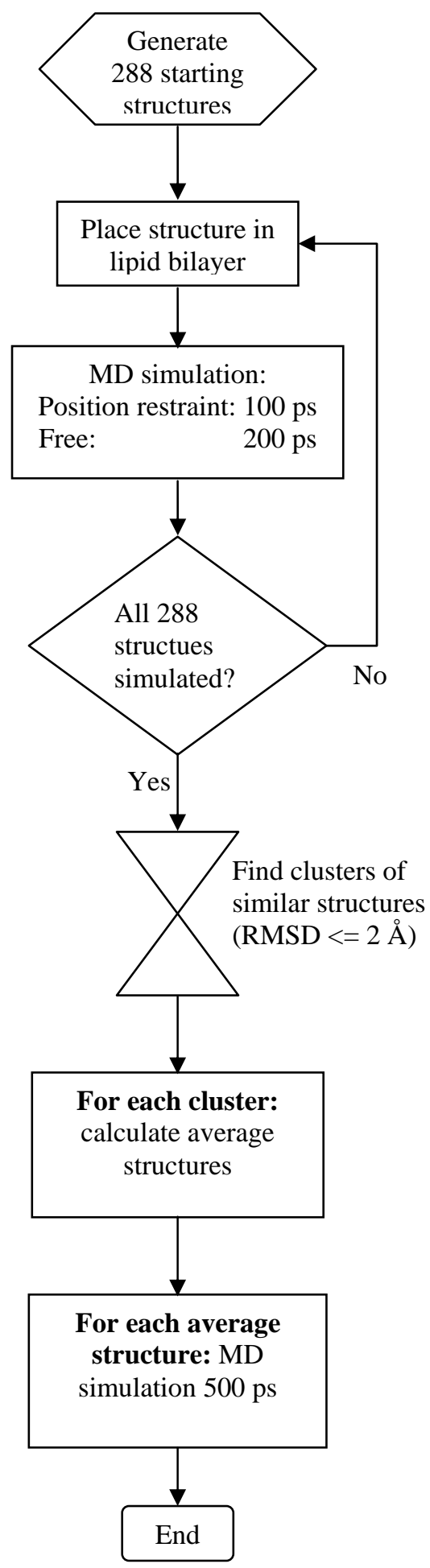

Figure 1 

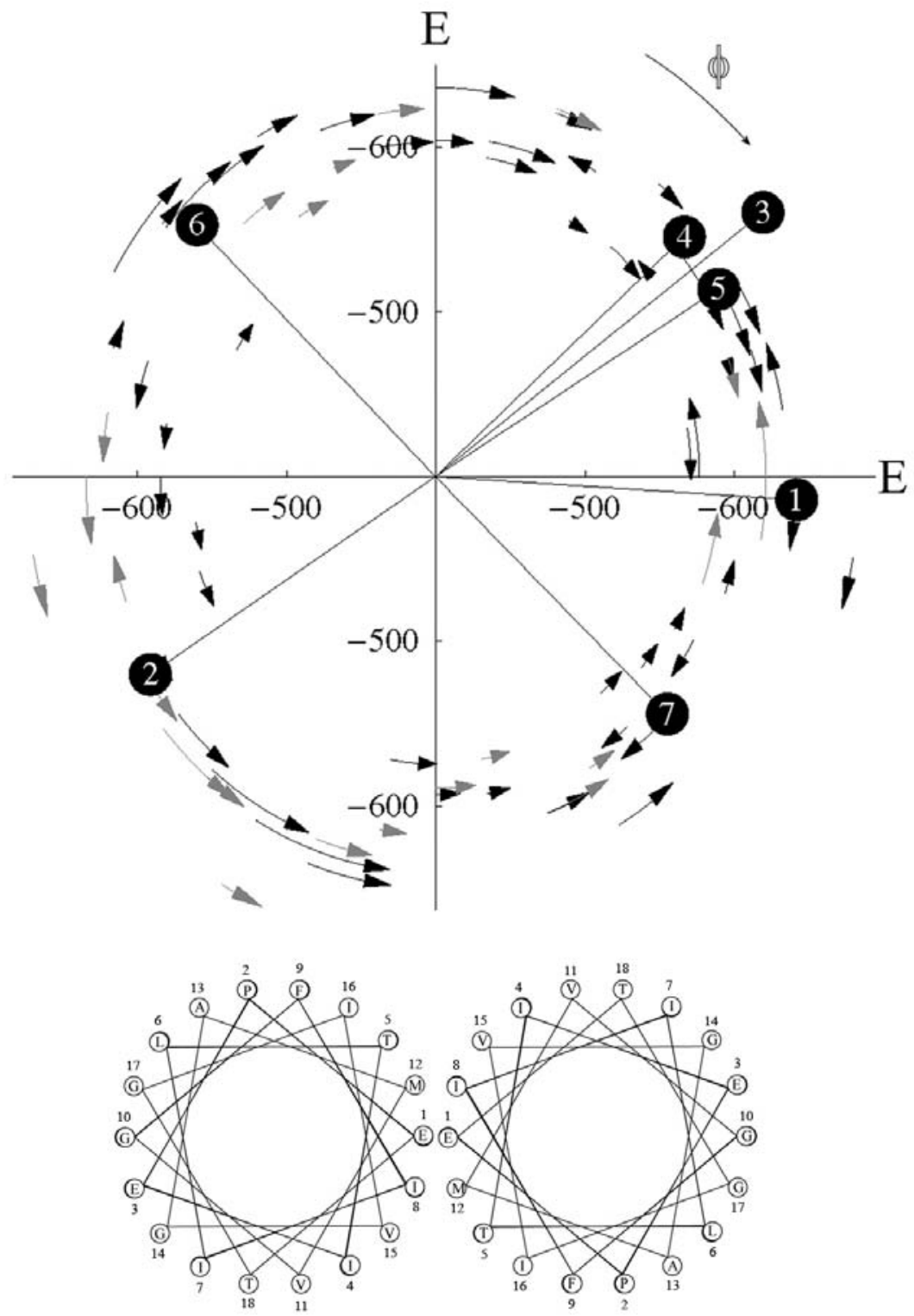

Figure 2 

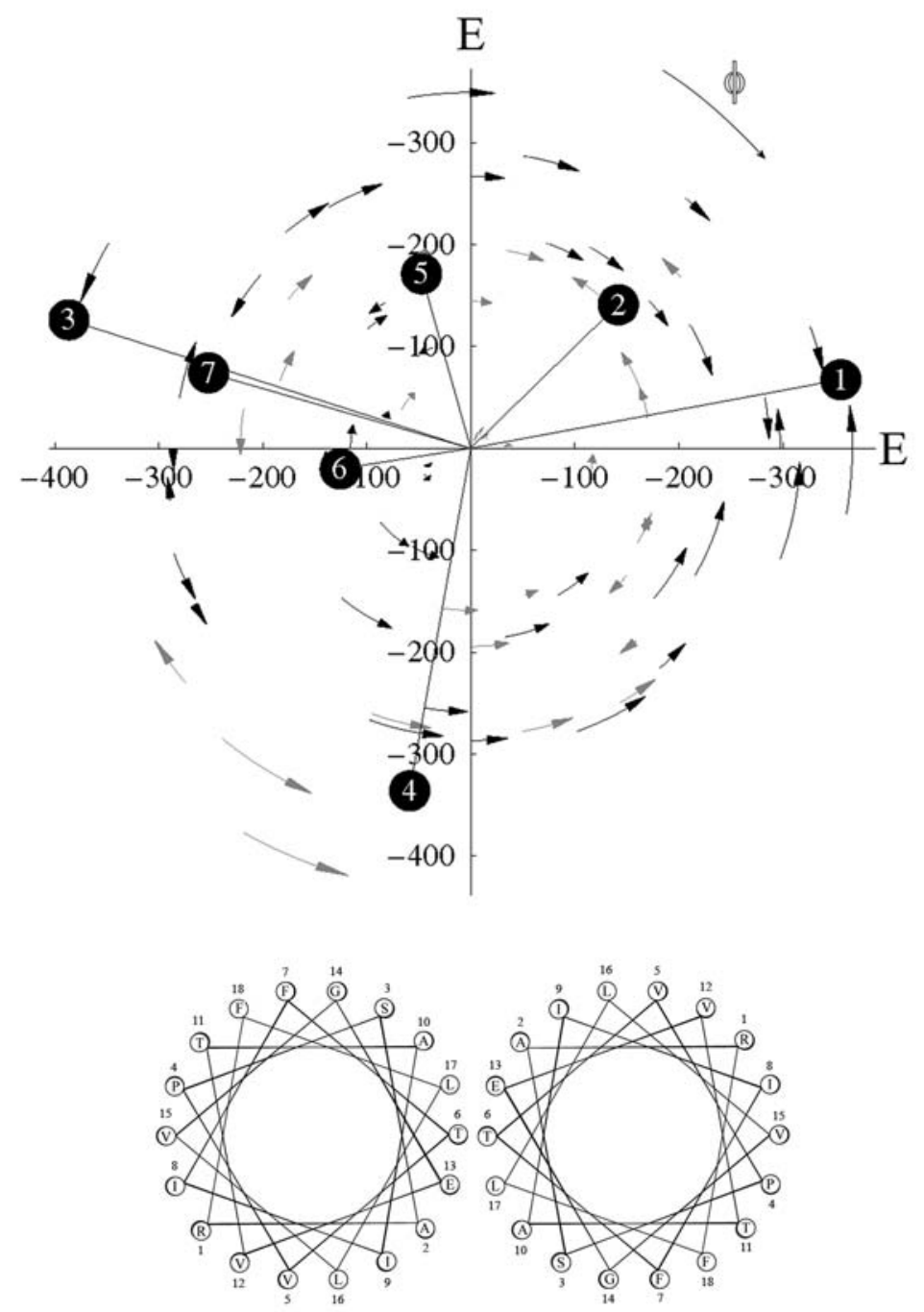

Figure 3 
a
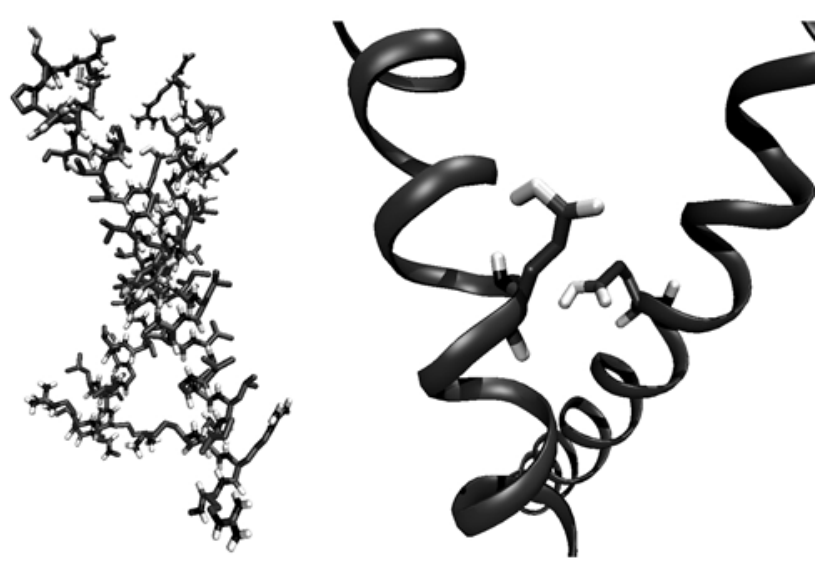

b
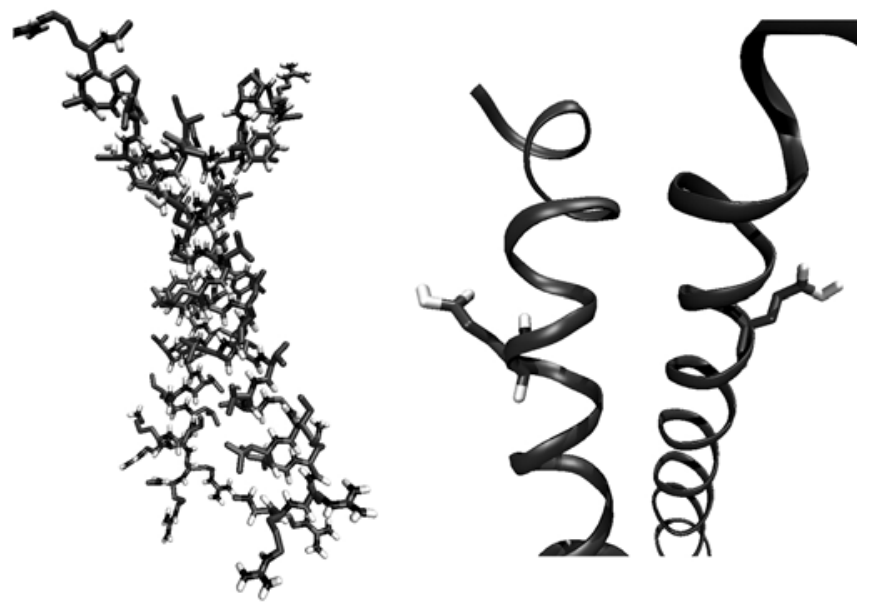

C
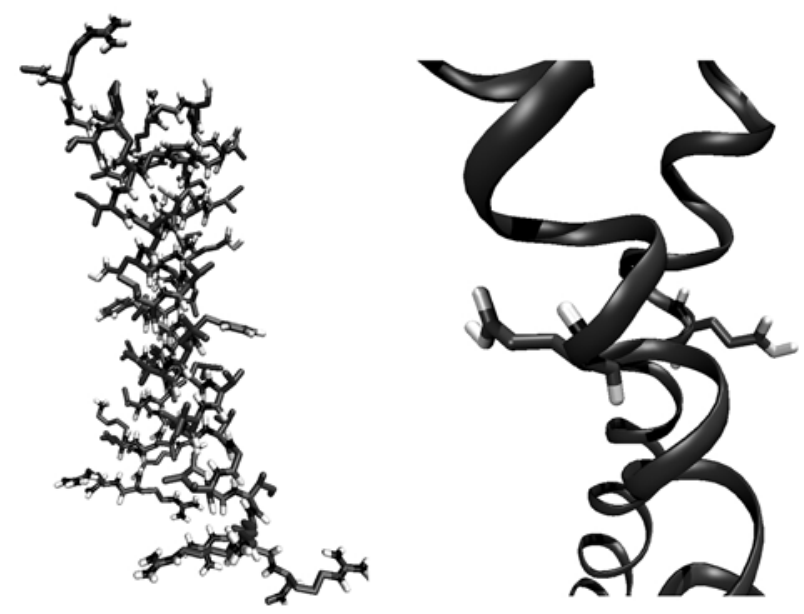

Figure 4 
Transmembrane molecular dynamics search 


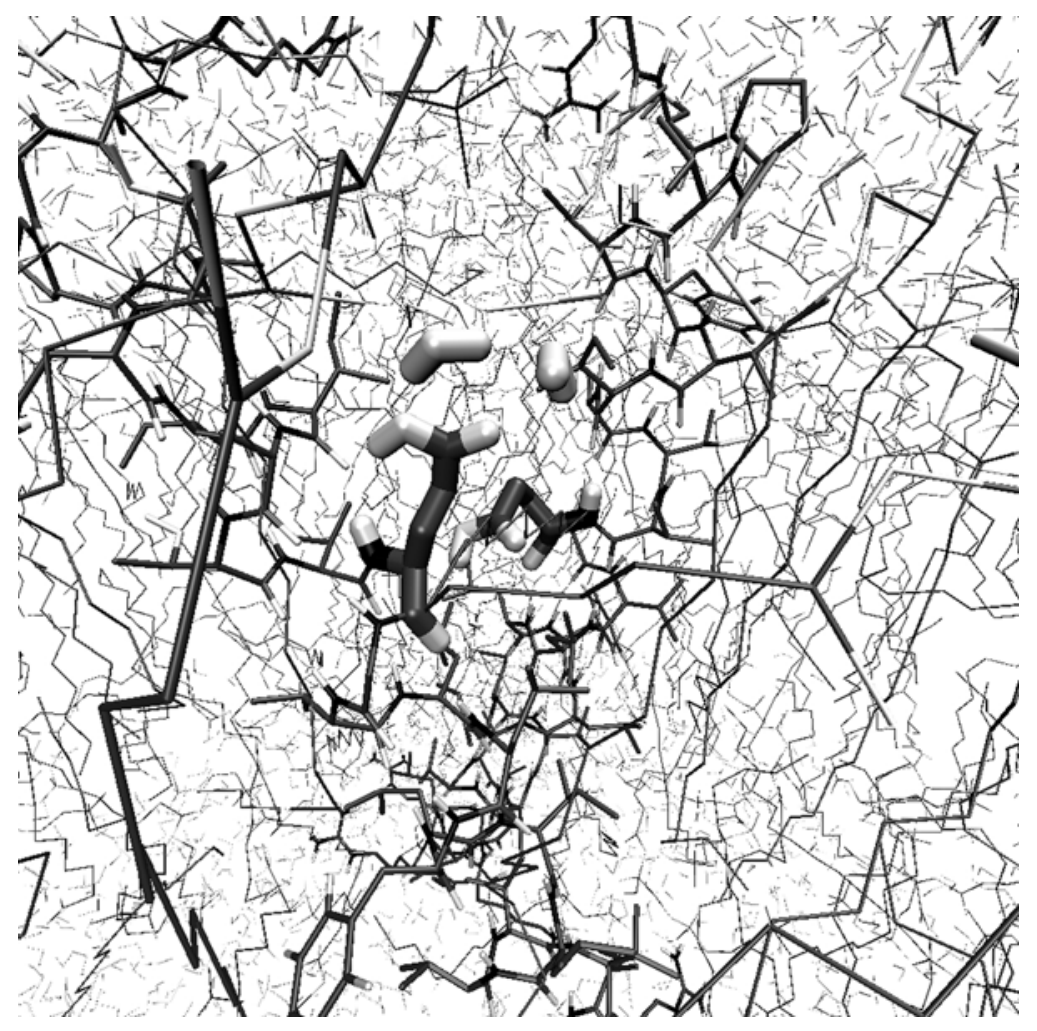

Figure 5 